\title{
New $F$ Expansion Method and Its Applications to Modified KdV Equation
}

\author{
Mohammad Ali Bashir ${ }^{1}$ \& Lama Abdulaziz Alhakim²,3 \\ ${ }^{1}$ Mathematics Department, Faculty of Science, Alnillin University, Alkortom, Alsodan \\ ${ }^{2}$ Mathematics Department, Faculty of post Graduate Studies, Omdurman Islamic University, Alkortom, Alsodan \\ 3 Department of Management Information System and Production Management, College of Business \& Eco- \\ nomics, Qassim University, Buraidah, K.S.A.
}

Correspondence: Lama Abdulaziz Alhakim, Mathematics Department, Faculty of post Graduate Studies, Omdurman Islamic University, Alkortom, Alsodan; Department of Management Information System and Production Management, College of Business \& Economics, Qassim University, Buraidah, K.S.A. E-mail: Lama2736@gmail.com

Received: September 25, 2013 Accepted: October 31, 2013 Online Published: November 20, 2013

doi:10.5539/jmr.v5n4p83 URL: http://dx.doi.org/10.5539/jmr.v5n4p83

\begin{abstract}
In this paper, the new $F$-expansion method is proposed for constructing more general exact solutions nonlinear evolution equation with the aid of symbolic computation. By using this method, we have successfully obtained some travelling wave solutions of the modified KdV Equation. These exact solutions include the hyperbolic function solutions, trigonometric function solutions, rational function solutions and Jacobi elliptic function solutions. Also it is shown that the proposed method is efficient for solving nonlinear evolution equations in mathematical physics and in engineering.
\end{abstract}

Keywords: F-Expansion method, modified KdV equation, exact solitons, travelling wave solution

\section{Introduction}

A lot of phenomena in physics and other domains are frequently described by nonlinear partial differential equations (NLPDEs). The study of exact solutions, especially traveling wave solutions, for NLPDEs plays a significant role in the study of nonlinear physical phenomena. These exact solutions when they exist can help to understand the dynamical processes that are modeled by the corresponding nonlinear evolution equations (NLEEs). The solitary wave is one of the basic nonlinear wave events appearing in fluid dynamics (Whitham, 1974) and plasma physics (Davidson, 1972; Ablowitz \& Clarkson, 1991).

Recently, the maple or Mathematica have been used efficiently to obtain exact analytical solutions to NLPDEs. Further, a number of direct approaches have been proposed to obtain traveling wave solutions to NLEEs among them the inverse scattering method (Ablowitz \& Clarkson, 1991), the tanh-function method (Malfliet, 2004), the extended tanh-function method (Fan, 2000) and the homogeneous balance method (Wang, 1996). It is assumed in this paper that the exact solution is expressed by a simple expansion $u(x, t)=u(\xi)=\sum_{i=0}^{n} A_{i} \cdot F^{i}(\xi)$. where $A_{i}$ are constants to be determined and the function $F(\xi)$ is defined by the solution of an auxiliary ordinary differential equation(ODE). The exact solutions expressed by a variety of Jacobi elliptic functions (JEFs) of many NLEEs have been obtained by Jacobi elliptic function expansion method (Liu, Fu, Liu, \& Zhao, 2001; Chen \& Zhang, 2003), mapping method (Peng, 2003; Peng, 2005), F expansion method (Zhao, Wang, \& Wang, 2003), extended F expansion method (Abdou, 2007; Liu \& Yang, 2004), the generalized Jacobi elliptic function method (Huiqun Zhang, 2007; Chen \& Zhang, 2004), and other methods (Elwakil, El-Labany, Zahran, \& Sabry, 2002; Khater, Hassan, Krishnan, \& Peng, 2008). Various exact solutions were obtained by using these methods, including the solitary wave solutions, shock wave solutions and periodic wave solutions.

\section{Summary of the Expansion Method}

Step 1 Use the transformation $u(x, t)=u(\xi) ; \xi=x-k t, k$ is an arbitrary constant, and reduce a given NLPDE, 
say in two independent variables,

$$
P\left(x, u_{t}, u_{x}, u_{t t}, u_{x x}, u_{x t}, \ldots \ldots\right)=0
$$

to the (ODE)

$$
H\left(u, u^{\prime}, u^{\prime \prime}, \ldots,\right)=0, \quad u^{\prime}=\frac{d u}{d \xi}
$$

In general, the left hand side of (1) is a polynomial in $u$ and its various derivatives.

Step 2 The $F$-expansion method gives the solution of (1) in the form

$$
u(x, t)=u(\xi)=\sum_{i=0}^{N} \alpha_{i} \cdot F^{i}(\xi)
$$

where $\alpha_{i}(i=0,1,2, \ldots, N)$ are constants to be determined and $F(\xi)$ satisfies the first order nonlinear ODE in the form

$$
\left(F^{\prime}(\xi)\right)^{2}=A_{4} F^{4}(\xi)+A_{3} F^{3}(\xi)+A_{2} F^{2}(\xi)+A_{1} F^{1}(\xi)+A_{0}
$$

where $A_{4}, A_{3}, A_{2}, A_{1}$, and $A_{0}$ are constants and $N$ in (3) can be obtained by balancing the nonlinear term(s) and the highest order derivatives in (1).

Step 3 Substituting the Equation (3) into (2) and using (4); setting coefficients of the polynomial to zero, we obtain a system of algebraic equations containing $\alpha_{0}, \alpha_{1}, \ldots, \alpha_{N}$ and $k$.

Step 4 Using Mathematica or Maple to solve these equations we obtain $\alpha_{0}, \alpha_{1}, \ldots, \alpha_{N}$ and $k$, which can be expressed by $A_{4}, A_{3}, A_{2}, A_{1}, A_{0}$.

Step 5 By substituting the obtained results into Equation (3), a general form of traveling wave solution of the NLPDE (1) is obtained. Several solutions of (4) have been well know for us (see Appendix A). Substituting the values of $A_{4}, A_{3}, A_{2}, A_{1}, A_{0}$ and the JEF solution $F(\xi)$ into the general form of solution, various classes of exact solutions of Equation (1) involving JEFs are obtained. Moreover, a short description of the mapping method to find the traveling wave solutions of Equation (1) in the form $u(x, t)=u(\xi) ; \xi=x-k t, k$ is an arbitrary constant, is given.

\section{Exact Solutions of the Modified KdV Equation}

In this section, we apply this method to construct the exact interaction solution solutions of the modified KdV equation $(\mathrm{mKdV})$

$$
u_{t}+\delta u^{2} u+u_{x x x}=0
$$

The transformation $u(x, t)=u(\xi) ; \xi=x-k t$ reduces Equation (5) to the ordinary differential equation (ODE):

$$
-3 k u+u^{3}+u^{\prime \prime}=0
$$

We can determine the positive integer $N$ by balancing $u^{3}$ and $u^{\prime \prime}$ in the given system equations. So we can suppose that Equation (6) has the following form:

$$
u(\xi)=\alpha_{0}+\alpha_{0} \cdot F(\xi)
$$

Substituting (7) into (6) and equating the coefficients of powers of $(F(\xi) \hat{F}(\xi))$ to zero, a system of algebraic equations are found. By resolving these system the following results are obtained:

$$
\alpha_{0}=\frac{-3 A_{3}}{\delta \sqrt{\frac{-6 A_{4}}{\delta}}}, \quad \alpha_{1}=\sqrt{\frac{-6 A_{4}}{\delta}}, \quad k=\left(\frac{2 A_{4} A_{2}-3 A_{3}^{2}}{2 A_{4}}\right)
$$

Substituting (8) into (7) yields

$$
u(\xi)=\frac{-3 A_{3}}{\delta \sqrt{\frac{-6 A_{4}}{\delta}}}+\sqrt{\frac{-6 A_{4}}{\delta}} \cdot F(\xi)
$$

where $\xi=x-\left(\frac{2 A_{4} A_{2}-3 A_{3}^{2}}{2 A_{4}}\right) t$. 
According to appendix A, we have the following families of exact solutions:

Family 1 If $A_{0}=1, A_{1}=0, A_{2}=-\left(1+m^{2}\right), A_{3}=0, A_{4}=m^{2}$, then we get

$$
u(\xi)=\sqrt{\frac{-6 m^{2}}{\delta}} \cdot \operatorname{sn}(\xi),
$$

where $\xi=x+\left(1+m^{2}\right) t$.

In particular setting $m=\frac{1}{2}, \delta=-6$.

we find:

$$
u(\xi)=\frac{1}{2} \cdot \operatorname{sn}(\xi)
$$

where $\xi=x+\left(\frac{5}{4}\right) t$ (See Figure 1).

Family 2 If $A_{0}=\left(1-m^{2}\right), A_{1}=0, A_{2}=\left(2 m^{2}-1\right), A_{3}=0, A_{4}=-m^{2}$, then we get

$$
u(\xi)=\sqrt{\frac{6 m^{2}}{\delta}} \cdot \operatorname{cn}(\xi),
$$

where $\xi=x-\left(2 m^{2}-1\right) t$.

In particular setting $m=\frac{1}{2}, \delta=-6$.

We find:

$$
u(\xi)=\frac{1}{2} \cdot c n(\xi)
$$

where $\xi=x+\left(\frac{5}{4}\right) t$ (See Figure 2).

Family 3 If $A_{0}=m^{2}-1, A_{1}=0, A_{2}=2-m^{2}, A_{3}=0, A_{4}=-1$, then we get

$$
u(\xi)=\sqrt{\frac{6}{\delta}} \cdot d n(\xi)
$$

where $\xi=x-\left(2-m^{2}\right) t$.

In particular setting $m=\frac{1}{2}, \delta=6$.

We find:

$$
u(\xi)=d n(\xi)
$$

where $\xi=x-\left(\frac{7}{4}\right) t$ (See Figure 3).

Family 4 If $A_{0}=m^{2}, A_{1}=0, A_{2}=-\left(m^{2}+1\right), A_{3}=0, A_{4}=1$, then we get

$$
u(\xi)=\sqrt{\frac{-6}{\delta}} \cdot n s(\xi),
$$

where $\xi=x-\left(m^{2}+1\right) t$.

In particular setting $m=\frac{1}{2}, \delta=-6$.

We find:

$$
u(\xi)=n s(\xi)
$$

where $\xi=x-\left(\frac{5}{4}\right) t$ (See Figure 4).

Family 5 If $A_{0}=-m^{2}, A_{1}=0, A_{2}=2 m^{2}-1, A_{3}=0, A_{4}=1-m^{2}$, then we get

$$
u(\xi)=\sqrt{\frac{-6\left(1-m^{2}\right)}{\delta}} \cdot n c(\xi),
$$


where $\xi=x-\left(2 m^{2}-1\right) t$.

In particular setting $m=\frac{1}{2}, \delta=6$.

We find:

$$
u(\xi)=\sqrt{\frac{3}{4}} n c(\xi),
$$

where $\xi=x+\left(\frac{1}{2}\right) t$ (See Figure 5).

Family 6 If $A_{0}=-1, A_{1}=0, A_{2}=2-m^{2}, A_{3}=0, A_{4}=m^{2}-1$, then we get

$$
u(\xi)=\sqrt{\frac{-6\left(m^{2}-1\right)}{\delta}} \cdot n d(\xi),
$$

where $\xi=x-\left(2-m^{2}\right) t$.

In particular setting $m=\frac{1}{2}, \delta=6$.

We find:

$$
u(\xi)=\sqrt{\frac{3}{4}} n d(\xi),
$$

where $\xi=x-\left(\frac{7}{4}\right) t$ (See Figure 6).

Family 7 If $A_{0}=1-m^{2}, A_{1}=0, A_{2}=2-m^{2}, A_{3}=0, A_{4}=1$, then we get

$$
u(\xi)=\sqrt{\frac{-6}{\delta}} \cdot \operatorname{cs}(\xi)
$$

where $\xi=x-\left(2-m^{2}\right) t$.

In particular setting $m=\frac{1}{2}, \delta=-6$.

We find:

$$
u(\xi)=c s(\xi)
$$

where $\xi=x-\left(\frac{7}{4}\right) t$ (See Figure 7).

Family 8 If $A_{0}=1, A_{1}=0, A_{2}=2-m^{2}, A_{3}=0, A_{4}=1-m^{2}$, then we get

$$
u(\xi)=\sqrt{\frac{-6\left(1-m^{2}\right)}{\delta}} \cdot s c(\xi),
$$

where $\xi=x-\left(2-m^{2}\right) t$.

In particular setting $m=\frac{1}{2}, \delta=-6$.

We find:

$$
u(\xi)=\frac{\sqrt{3}}{4} s c(\xi)
$$

where $\xi=x-\left(\frac{7}{4}\right) t$ (See Figure 8).

Family 9 If $A_{0}=1, A_{1}=0, A_{2}=2 m^{2}-1, A_{3}=0, A_{4}=m^{2}\left(m^{2}-1\right)$, then we get

$$
u(\xi)=\sqrt{\frac{-6 m^{2}\left(m^{2}-1\right)}{\delta}} \cdot s d(\xi),
$$

where $\xi=x-\left(2 m^{2}-1\right) t$.

In particular setting $m=\frac{1}{2}, \delta=6$.

We find:

$$
u(\xi)=s d(\xi),
$$


where $\xi=x+\left(\frac{1}{2}\right) t$ (See Figure 9).

Family 10 If $A_{0}=m^{2}\left(m^{2}-1\right), A_{1}=0, A_{2}=2 m^{2}-1, A_{3}=0, A_{4}=1$, then we get

$$
u(\xi)=\sqrt{\frac{-6}{\delta}} \cdot d s(\xi),
$$

where $\xi=x-\left(2 m^{2}-1\right) t$.

In particular setting $m=\frac{1}{2}, \delta=-6$.

We find:

$$
u(\xi)=d s(\xi)
$$

where $\xi=x+\left(\frac{1}{2}\right) t$ (See Figure 10).

Family 11 If $A_{0}=\frac{1}{4}, A_{1}=0, A_{2}=\frac{1}{2}\left(1-2 m^{2}\right), A_{3}=0, A_{4}=\frac{1}{4}$, then we get

$$
u(\xi)=\sqrt{\frac{-3}{2 \cdot \delta}} \cdot(n s(\xi)+c s(\xi)),
$$

where $\xi=x-\frac{1}{2}\left(1-2 m^{2}\right) t$.

In particular setting $m=\frac{1}{2}, \delta=-6$.

We find:

$$
u(\xi)=\frac{1}{2}(n s(\xi)+c s(\xi))
$$

where $\xi=x-\left(\frac{1}{4}\right) t$ (See Figure 11).

Family 12 If $A_{0}=\frac{1}{4}\left(1-m^{2}\right), A_{1}=0, A_{2}=\frac{1}{2}\left(1+m^{2}\right), A_{3}=0, A_{4}=\frac{1}{4}\left(1-m^{2}\right)$, then we get

$$
u(\xi)=\sqrt{\frac{-3 \cdot\left(1-m^{2}\right)}{2 \cdot \delta}} \cdot(n s(\xi)+s c(\xi)),
$$

where $\xi=x-\frac{1}{2}\left(1+m^{2}\right) t$.

In particular setting $m=\frac{1}{2}, \delta=-6$.

We find:

$$
u(\xi)=\frac{\sqrt{3}}{4}(n s(\xi)+s c(\xi)),
$$

where $\xi=x-\left(\frac{5}{8}\right) t$ (See Figure 12).

Family 13 If $A_{0}=\frac{m^{2}}{4}, A_{1}=0, A_{2}=\frac{1}{2}\left(m^{2}-2\right), A_{3}=0, A_{4}=\frac{1}{4}$, then we get

$$
u(\xi)=\sqrt{\frac{-3}{2 \cdot \delta}} \cdot(n s(\xi)+d s(\xi))
$$

where $\xi=x-\frac{1}{2}\left(m^{2}-2\right) t$.

In particular setting $m=\frac{1}{2}, \delta=-6$.

We find:

$$
u(\xi)=\frac{1}{2}(n s(\xi)+d s(\xi))
$$

where $\xi=x+\left(\frac{7}{8}\right) t$ (See Figure 13).

Family 14 If $A_{0}=\frac{m^{2}}{4}, A_{1}=0, A_{2}=\frac{1}{2}\left(m^{2}-2\right), A_{3}=0, A_{4}=\frac{m^{2}}{4}$, then we get

$$
u(\xi)=\sqrt{\frac{-3 m^{2}}{4 \cdot \delta}} \cdot \frac{d n(\xi)}{\sqrt{1-m^{2}} \operatorname{sn}(\xi)+c n(\xi)},
$$


where $\xi=x-\frac{1}{2}\left(m^{2}-2\right) t$.

In particular setting $m=\frac{1}{2}, \delta=-6$.

We find:

$$
u(\xi)=\frac{1}{4} \frac{d n(\xi)}{\sqrt{\frac{3}{4}} \operatorname{sn}(\xi)+c n(\xi)},
$$

where $\xi=x+\left(\frac{7}{8}\right) t$ (See Figure 14).

Family 15 If $A_{0}=-\frac{1}{4}\left(1-m^{2}\right)^{2}, A_{1}=0, A_{2}=\frac{1}{2}\left(1+m^{2}\right), A_{3}=0, A_{4}=-\frac{1}{4}$, then we get

$$
u(\xi)=\sqrt{\frac{3}{2 \cdot \delta}} \cdot(\operatorname{mcn}(\xi)+d n(\xi))
$$

where $\xi=x-\frac{1}{2}\left(1+m^{2}\right) t$.

In particular setting $m=\frac{1}{2}, \delta=6$.

We find:

$$
u(\xi)=\frac{1}{2}\left(\frac{1}{2} \operatorname{cn}(\xi)+d n(\xi)\right)
$$

where $\xi=x-\left(\frac{5}{8}\right) t$ (See Figure 15).

Family 16 If $A_{0}=\frac{1}{4}, A_{1}=0, A_{2}=\frac{1}{2}\left(1-2 m^{2}\right), A_{3}=0, A_{4}=\frac{1}{4}$, then we get

$$
u(\xi)=\sqrt{\frac{-3}{2 \cdot \delta}} \cdot\left(\frac{s n(\xi)}{1+c n(\xi)}\right)
$$

where $\xi=x-\frac{1}{2}\left(1-2 m^{2}\right) t$.

In particular setting $m=\frac{1}{2}, \delta=-6$.

We find:

$$
u(\xi)=\frac{1}{2}\left(\frac{\operatorname{sn}(\xi)}{1+c n(\xi)}\right)
$$

where $\xi=x-\left(\frac{1}{4}\right) t$ (See Figure 16).

Family 17 If $A_{0}=\frac{m^{2}}{4}, A_{1}=0, A_{2}=\frac{1}{2}\left(1-2 m^{2}\right), A_{3}=0, A_{4}=\frac{1}{4 m^{2}}$, then we get

$$
u(\xi)=\sqrt{\frac{-3}{2 \cdot \delta \cdot m^{2}}} \cdot\left(\frac{d n(\xi)}{\sqrt{\frac{1-m^{2}}{m^{2}}}+c n(\xi)}\right),
$$

where $\xi=x-\frac{1}{2}\left(1-2 m^{2}\right) t$.

In particular setting $m=\frac{1}{2}, \delta=-6$.

We find:

$$
u(\xi)=\left(\frac{d n(\xi)}{\sqrt{3}+c n(\xi)}\right)
$$

where $\xi=x-\left(\frac{1}{4}\right) t$ (See Figure 17).

Family 18 If $A_{0}=\frac{1}{4}, A_{1}=0, A_{2}=\frac{1}{2}\left(m^{2}-2\right), A_{3}=0, A_{4}=\frac{m^{2}}{4}$, then we get

$$
u(\xi)=\sqrt{\frac{-3}{2 \cdot \delta \cdot m^{2}}} \cdot\left(\frac{s n(\xi)}{1+d n(\xi)}\right),
$$


Where $\xi=x-\frac{1}{2}\left(m^{2}-2\right) t$.

In particular setting $m=\frac{1}{2}, \delta=-6$.

We find:

$$
u(\xi)=\left(\frac{\operatorname{sn}(\xi)}{1+d n(\xi)}\right)
$$

where $\xi=x+\left(\frac{7}{8}\right) t$ (See Figure 18).

Family 19 If $A_{0}=\frac{1}{4}\left(m^{2}-1\right), A_{1}=0, A_{2}=\frac{1}{2}\left(1+m^{2}\right), A_{3}=0, A_{4}=\frac{1}{4}\left(1-m^{2}\right)$, then we get

$$
u(\xi)=\sqrt{\frac{-6\left(1-m^{2}\right)}{\delta}} \cdot\left(\frac{d n(\xi)}{1+m s n(\xi)}\right),
$$

where $\xi=x-\frac{1}{2}\left(1+m^{2}\right) t$.

In particular setting $m=\frac{1}{2}, \delta=-6$.

We find:

$$
u(\xi)=\left(\frac{d n(\xi)}{1+\frac{1}{2} \cdot \operatorname{sn}(\xi)}\right)
$$

where $\xi=x-\left(\frac{5}{8}\right) t$ (See Figure 19).

Family 20 If $A_{0}=\frac{1}{4}\left(1-m^{2}\right), A_{1}=0, A_{2}=\frac{1}{2}\left(1+m^{2}\right), A_{3}=0, A_{4}=\frac{1}{4}\left(1-m^{2}\right)$, then we get

$$
u(\xi)=\sqrt{\frac{-3\left(1-m^{2}\right)}{2 \cdot \delta}} \cdot\left(\frac{c n(\xi)}{1+\operatorname{sn}(\xi)}\right),
$$

where $\xi=x-\frac{1}{2}\left(1+m^{2}\right) t$.

In particular setting $m=\frac{1}{2}, \delta=-6$.

We find:

$$
u(\xi)=\frac{\sqrt{3}}{4}\left(\frac{c n(\xi)}{1+\operatorname{sn}(\xi)}\right)
$$

where $\xi=x-\left(\frac{5}{8}\right) t$ (See Figure 20).

Family 21 If $A_{0}=\frac{1}{4}, A_{1}=0, A_{2}=\frac{1}{2}\left(1+m^{2}\right), A_{3}=0, A_{4}=\frac{1}{4}\left(1-m^{2}\right)^{2}$, then we get

$$
u(\xi)=\sqrt{\frac{-3\left(1-m^{2}\right)^{2}}{2 \cdot \delta}} \cdot\left(\frac{s n(\xi)}{\operatorname{cn}(\xi)+d n(\xi)}\right),
$$

where $\xi=x-\frac{1}{2}\left(1+m^{2}\right) t$.

In particular setting $m=\frac{1}{2}, \delta=-6$.

We find:

$$
u(\xi)=\frac{3}{8}\left(\frac{s n(\xi)}{\operatorname{cn}(\xi)+d n(\xi)}\right)
$$

where $\xi=x-\left(\frac{5}{8}\right) t$ (See Figure 21).

Family 22 If $A_{0}=\frac{1}{4}, A_{1}=0, A_{2}=\frac{1}{2}\left(2-m^{2}\right), A_{3}=0, A_{4}=\frac{m^{4}}{4}$, then we get

$$
u(\xi)=\sqrt{\frac{-3 m^{4}}{2 \cdot \delta}} \cdot\left(\frac{c n(\xi)}{\sqrt{1-m^{2}}+\operatorname{dn}(\xi)}\right),
$$

where $\xi=x-\frac{1}{2}\left(2-m^{2}\right) t$. 
In particular setting $m=\frac{1}{2}, \delta=-6$.

We find:

$$
u(\xi)=\frac{1}{8}\left(\frac{c n(\xi)}{\sqrt{\frac{3}{4}}+d n(\xi)}\right)
$$

where $\xi=x-\left(\frac{7}{8}\right) t$ (See Figure 22).

Family 23 If $A_{0}=0, A_{1}=0, A_{2}=-1, A_{3}=A_{3}, A_{4}=A_{4}$, then we get

$$
u(\xi)=\frac{-3 A_{3}}{\delta \sqrt{\frac{-6 A_{4}}{\delta}}}+\sqrt{\frac{-6 A_{4}}{\delta}} \cdot \frac{\left(A_{3}+A_{3} \tan ^{2}(\xi-c)+\sqrt{\tan ^{2}(\xi-c)\left(1+\tan ^{2}(\xi-c)\right)\left(A_{3}^{2}+4 A_{4}\right)}\right)}{\left(\frac{A_{3}^{2}-4 A_{4} \tan ^{2}(\xi-c)}{2}\right)},
$$

where $\xi=x+\left(\frac{2 A_{4}+3 A_{3}^{2}}{2 A_{4}}\right) t$.

In particular setting $\delta=-6, c=0, A_{3}=1, A_{4}=1$.

We find:

$$
u(\xi)=\frac{1}{2}-2\left(\frac{1+\tan ^{2}(\xi)+\sqrt{5} \cdot \sqrt{\tan ^{2}(\xi)\left(1+\tan ^{2}(\xi)\right)}}{4 \tan ^{2}(\xi)-1}\right),
$$

where $\xi=x+\left(\frac{5}{2}\right) t$ (See Figure 23).

Family 24 If $A_{0}=0, A_{1}=0, A_{2}=0, A_{3}=A_{3}, A_{4}=A_{4}$, then we get

$$
u(\xi)=\frac{-3 A_{3}}{\delta \sqrt{\frac{-6 A_{4}}{\delta}}}+\sqrt{\frac{-6 A_{4}}{\delta}} \cdot\left(\frac{4 A_{3}}{A_{3}^{2} \xi^{2}-2 A_{3}^{2} c \xi+c^{2} A_{3}^{2}-4 A_{4}}\right),
$$

where $\xi=x+\left(\frac{-3 A_{3}^{2}}{2 A_{4}}\right) t$.

In particular setting $A_{3}=1, A_{4}=1, c=1, \delta=-6$.

We find:

$$
u(\xi)=\frac{1}{2}+\left(\frac{4}{\xi^{2}-2 \xi-3}\right)
$$

where $\xi=x-\left(\frac{3}{2}\right) t$ (See Figure 24).

Family 25 If $A_{0}=0, A_{1}=0, A_{2}=A_{2}, A_{3}=A_{3}, A_{4}=A_{4}$, then we get

$$
u(\xi)=\frac{-3 A_{3}}{\delta \sqrt{\frac{-6 A_{4}}{\delta}}}+\sqrt{\frac{-6 A_{4}}{\delta}} \cdot\left(\frac{4 A_{2} e^{\left(\sqrt{A_{2}}(\xi-c)\right)}}{4 A_{4} A_{2}-A_{3}^{2}-2 A_{3} e^{\left(\sqrt{A_{2}}(\xi-c)\right)}-2 A_{3} e^{\left(2 \sqrt{A_{2}}(\xi-c)\right)}}\right),
$$

where $\xi=x+\left(\frac{2 A_{4} A_{2}-3 A_{3}^{2}}{2 A_{4}}\right) t$.

In particular setting $\delta=-6, c=0, A_{3}=1, A_{4}=1$.

We find:

$$
u(\xi)=\frac{1}{2}+\left(\frac{4 e^{\xi}}{e^{2 \xi}-e^{\xi}-3}\right)
$$

where $\xi=x-\left(\frac{3}{2}\right) t$ (See Figure 25).

Family 26 If $A_{0}=-k, A_{1}=-k, A_{2}=0, A_{3}=k, A_{4}=k$, then we get

$$
u(\xi)=\frac{-3 k}{\delta \sqrt{\frac{-6 k}{\delta}}}+\sqrt{\frac{-6 k}{\delta}} \cdot\left(\frac{-i \cdot \sqrt{3}+s n^{2}\left(\frac{\sqrt{-i \cdot k \sqrt{3}}}{2} \xi, i\right)}{i \cdot \sqrt{3}+s n^{2}\left(\frac{\sqrt{-i \cdot k \sqrt{3}}}{2} \xi, i\right)},\right.
$$


or

$$
u(\xi)=\frac{-3 k}{\delta \sqrt{\frac{-6 k}{\delta}}}+\sqrt{\frac{-6 k}{\delta}} \cdot\left(\frac{\sqrt{3}+i \cdot s^{2}\left(\frac{\sqrt{-i \cdot k \sqrt{3}}}{2} \xi, i\right)}{-\sqrt{3}+i \cdot s^{2}\left(\frac{\sqrt{-i \cdot k \sqrt{3}}}{2} \xi, i\right)}\right),
$$

where $\xi=x+\left(\frac{-3 k}{2}\right) t$.

Remark Using Maple, we have verified all solutions we obtained by butting them back into the original equations.

\section{Table Graphics}

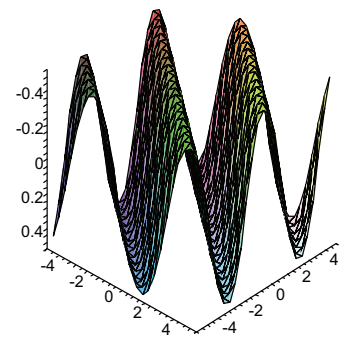

Figure 1. $u(\xi)=\frac{1}{2} \cdot \operatorname{sn}(\xi)$

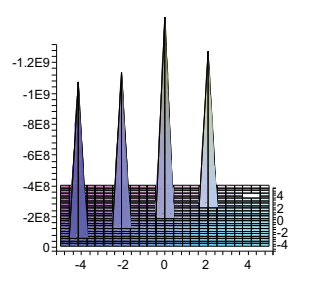

Figure 4. $u(x)=n s(\xi)$

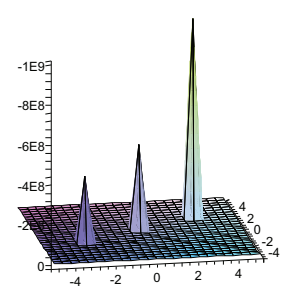

Figure 7. $u(\xi)=c s(\xi)$

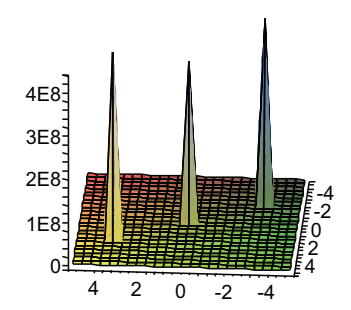

Figure 10. $u(\xi)=d s(\xi)$

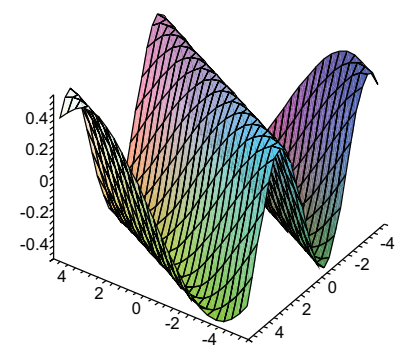

Figure 2. $u(\xi)=\frac{1}{2} \cdot c n(\xi)$

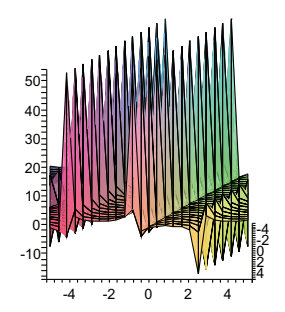

Figure 5. $u(\xi)=\sqrt{\frac{3}{4}} n c(\xi)$

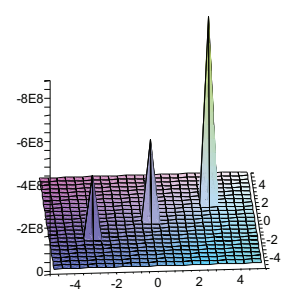

Figure 8. $u(\xi)=\frac{\sqrt{3}}{4} s c(\xi)$

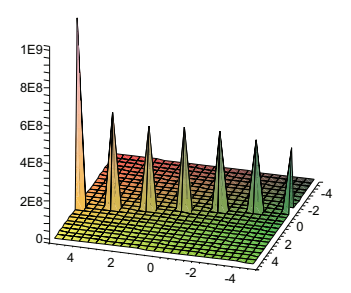

Figure 11. $u(\xi)=\frac{1}{2}(n s(\xi)+c s(\xi))$

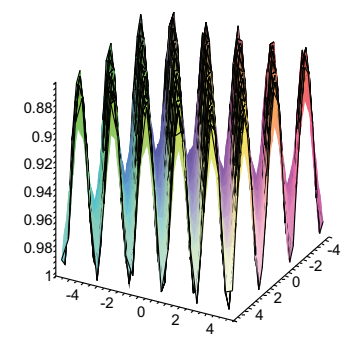

Figure 3. $u(\xi)=d n(\xi)$

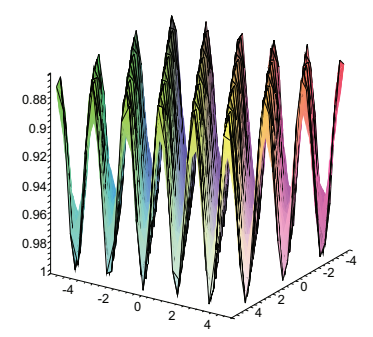

Figure 6. $u(\xi)=\sqrt{\frac{3}{4}} n d(\xi)$

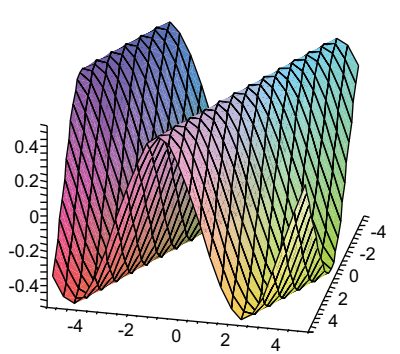

Figure 9. $u(\xi)=s d(\xi)$

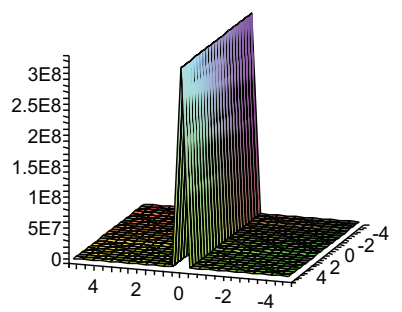

Figure 12. $u(\xi)=\frac{\sqrt{3}}{4}(n s(\xi)+s c(\xi))$ 


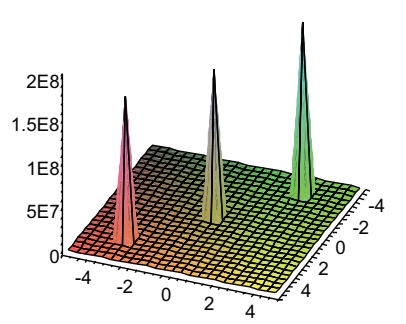

Figure13. $u(\xi)=\frac{1}{2}(n s(\xi)+d s(\xi))$

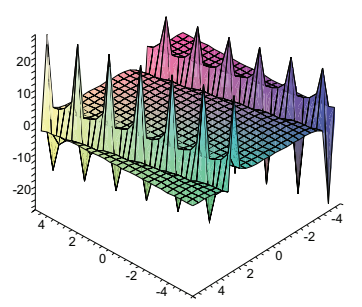

Figure 16. $u(\xi)=\frac{1}{2}\left(\frac{\operatorname{sn}(\xi)}{1+\operatorname{cn}(\xi)}\right)$

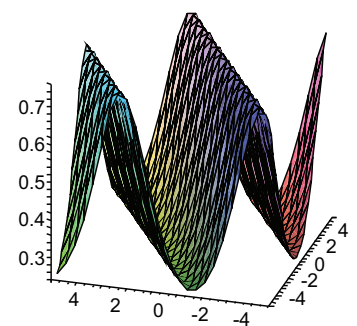

Figure 19. $u(\xi)=\left(\frac{d n(\xi)}{1+\frac{1}{2} \cdot \operatorname{sn}(\xi)}\right)$

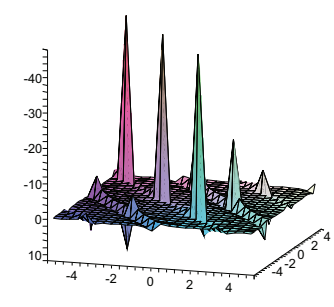

Figure 14. $u(\xi)=\frac{1}{4} \frac{d n(\xi)}{\sqrt{\frac{3}{4}} \operatorname{sn}(\xi)+c n(\xi)}$

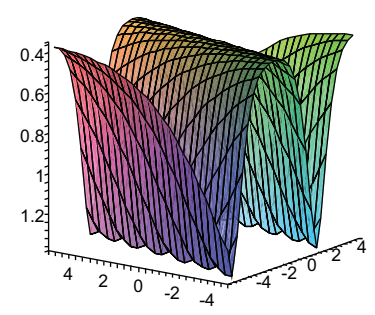

Figure 17. $u(\xi)=\left(\frac{d n(\xi)}{\sqrt{3}+c n(\xi)}\right)$

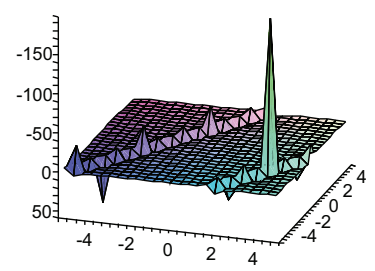

Figure 20. $u(\xi)=\frac{\sqrt{3}}{4}\left(\frac{c n(\xi)}{1+\operatorname{sn}(\xi)}\right)$

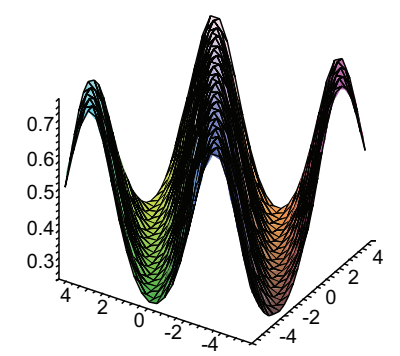

Figure15. $u(\xi)=\frac{\left(\frac{1}{2} c n(\xi)+d n(\xi)\right)}{2}$

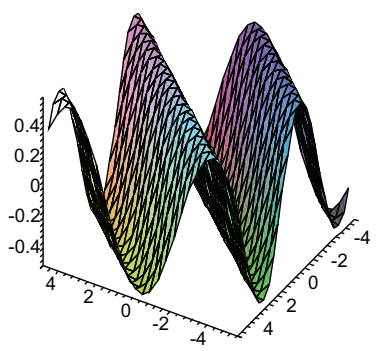

Figure 18. $u(\xi)=\left(\frac{s n(\xi)}{1+d n(\xi)}\right)$

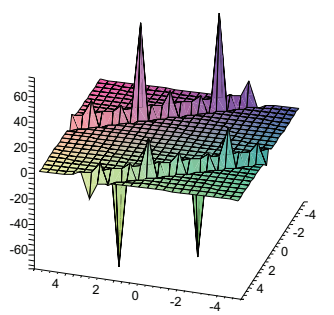

Figure 21. $u(\xi)=\frac{3}{8}\left(\frac{s n(\xi)}{c n(\xi)+d n(\xi)}\right)$

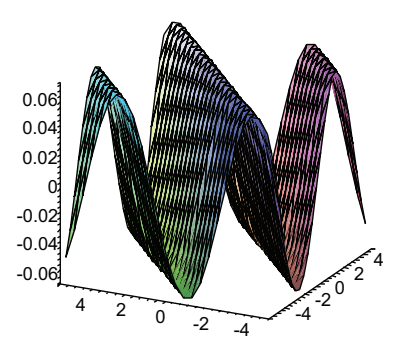

Figure 22. $u(\xi)=\frac{1}{8}\left(\frac{c n(\xi)}{\sqrt{\frac{3}{4}}+d n(\xi)}\right)$

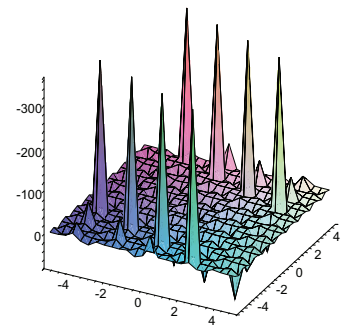

Figure 23. $u(\xi)=\frac{1}{2}-2\left(\frac{1+\tan ^{2}(\xi)+\sqrt{5} \cdot \sqrt{\tan ^{2}(\xi)\left(1+\tan ^{2}(\xi)\right)}}{4 \tan ^{2}(\xi)-1}\right)$ 


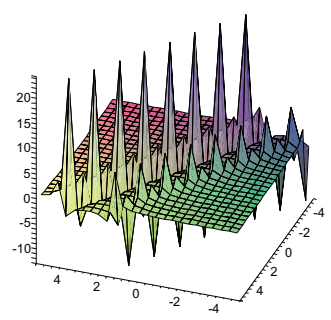

Figure 24. $u(\xi)=\frac{1}{2}+\left(\frac{4}{\xi^{2}-2 \xi-3}\right)$

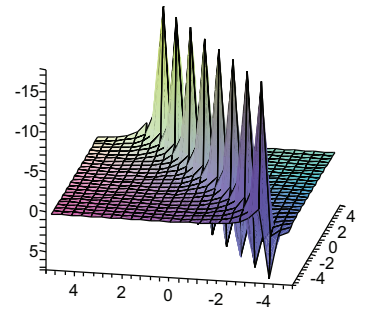

Figure 25. $u(\xi)=\frac{1}{2}+\left(\frac{4 e^{\xi}}{e^{2 \xi}-e^{\xi}-3}\right)$

\section{Conclusion}

The main idea of the $F$-expansion method is that the traveling wave solutions of nonlinear partial differential equations can be expressed as a polynomial in $F(\xi)$, where $F(\xi)$ satisfies the Equation (4) to some nonlinear PDEs in mathematical physics via the modified $\mathrm{KdV}$ equation.

We have obtained families of exact solutions of these equations in terms of Jacobi elliptic functions and hyperbolic functions Finally, we conclude according to the Appendix B that our results in terms of Jacobi elliptic functions generate into hyperbolic functions when $m \rightarrow 1$ and generate into trigonometric functions when $m \rightarrow 0$.

\section{References}

Abdou, M. A. (2007). The extended F-expansion method and its application for a class of nonlinear evolution equations. Chaos, Solitons and Fractals, 31(1), 95-104. http://dx.doi.org/10.1016/j.chaos.2005.09.030

Ablowitz, M. J., \& Clarkson, P. A. (1991). Solitons, Nonlinear Evolution Equations and Inverseb Scattering Transform. Cambridge: Cambridge University Press. http://dx.doi.org/10.1017/CBO9780511623998

Chen, H. T., \& Zhang, H. Q. (2003). Improved Jacobin elliptic method and its applications. Chaos, Solitons and Fractals, 15, 585-591. http://dx.doi.org/10.1016/S0960-0779(02)00147-9

Chen, H. T., \& Zhang, H. Q. (2004). New double periodic and multiple soliton solutions of the generalized (2+1)dimensional Boussinesq equation. Chaos, Solitons and Fractals, 20, 765-769. http://dx.doi.org/10.1016/j.chaos.2003.08.006

Davidson, R. (1972). Methods in Nonlinear Plasma Theory. New York: Academic Press.

Elwakil, S. A., El-labany, S. K., Zahran, M. A., \& Sabry, R. (2002). Modified extended tanh-function method for solving nonlinear partial differential equations. Phys. Lett. A, 299, 179-188. http://dx.doi.org/10.1016/S0375-9601(02)00669-2

Fan, E., \& Zhang, H. (1998). A note on the homogeneous balance method. Phys. Lett. A, 246, 403-406. http://dx.doi.org/10.1016/S0375-9601(98)00547-7

Fan, E. G. (2000). Extended tanh-function method and its applications to nonlinear equations. Phys. Lett. A, 277, 212-218. http://dx.doi.org/10.1016/S0375-9601(00)00725-8

Khater, A. H., Hassan, M. M., Krishnan, E. V., \& Peng, Y. Z. (2008). Applications of elliptic functions to ionacoustic plasma waves. Eur. Phys. J. D, 50, 177-184. http://dx.doi.org/10.1140/epjd/e2008-00205-1

Liu, J., \& Yang, K. (2004). The extended F-expansion method and exact solutions of nonlinear PDEs. Chaos, Solitons and Fractals, 22, 111-121. http://dx.doi.org/10.1016/j.chaos.2003.12.069

Liu, S. K., Fu, Z. T., Liu, S. D., \& Zhao, Q. (2001). Jacobi elliptic function expansion method and periodic wave solutions of non linear wave equations. Phys. Lett. A, 289, 69-74. http://dx.doi.org/10.1016/S0375-9601(01)00580-1

Malfliet, W. (2004). The tanh method: a tool for solving certain classes of nonlinear evolution and wave equations. J. Comput. Appl. Math., 529-541. http://dx.doi.org/10.1016/S0377-0427(03)00645-9

Peng, Y. (2003). Exact periodic wave solutions to a new Hamiltonian amplitude equation. J. Phys. Soc. Japan, 72, 1356-1359. http://dx.doi.org/10.1143/JPSJ.72.1356 
Peng, Y. (2005). Exact periodic wave solutions to the Melnikov equation. Z. Naturforsch A, 60, 321-327.

Wang, M. (1996). Exact solutions for a compound KdV-Burgers equation. Phys. Lett. A, 213, $279-287$. http://dx.doi.org/10.1016/0375-9601(96)00103-X

Whitham, G. (2002). Linear and Nonlinear Waves. New York: Wiley.

Yang, Y., Tao, Z. L., \& Austin, R. F. (2010). Solutions of the generalized KdV equation with time dependent damping and dispersion. Appl. Math. Comput., 216, 1029-1035. http://dx.doi.org/10.1016/j.amc.2009.12.059

Zayed, E. M. E., \& Abdelaziz, M. A. M. (2011). The modified sine-cosine method and its applications to the generalized $\mathrm{K}(\mathrm{n}, \mathrm{n})$ and BBM equations with variable coefficients. International Journal of Nonlinear Science, 12(1), 95-99.

Zhang, H. Q. (2007). Extended Jacobi elliptic function expansion method and its applications. Communications in Nonlinear Science and Numerical Simulation, 5, 627-635. http://dx.doi.org/10.1016/j.cnsns.2005.08.003

Zhao, Y. B., Wang, Y. B., \& Wang, Y. M. (2003). Periodic wave solutions to a coupled KdV equations with variable coefficients. Phys. Lett. A, 308, 31-36. http://dx.doi.org/10.1016/S0375-9601(02)01775-9 


\section{Appendix A}

The general solutions to the Equation (4) are listed as follows:

\begin{tabular}{|c|c|c|c|c|c|}
\hline$A_{0}$ & $A_{1}$ & $A_{2}$ & $A_{3}$ & $A_{4}$ & $F(\xi)$ \\
\hline 1 & 0 & $\left(-1-m^{2}\right)$ & 0 & $m^{2}$ & $\operatorname{sn}(\xi)$ \\
\hline $1-m^{2}$ & 0 & $2 m^{2}-1$ & 0 & $-m^{2}$ & $c n(\xi)$ \\
\hline$m^{2}-1$ & 0 & $2-m^{2}$ & 0 & -1 & $d n(\xi)$ \\
\hline$m^{2}$ & 0 & $\left(-1-m^{2}\right)$ & 0 & 1 & $n s(\xi)$ \\
\hline$-m^{2}$ & 0 & $2 m^{2}-1$ & 0 & $1-m^{2}$ & $n c(\xi)$ \\
\hline-1 & 0 & $2-m^{2}$ & 0 & $m^{2}-1$ & $n d(\xi)$ \\
\hline 1 & 0 & $2-m^{2}$ & 0 & $1-m^{2}$ & $s c(\xi)$ \\
\hline 1 & 0 & $2 m^{2}-1$ & 0 & $\left(m^{4}-m^{2}\right)$ & $s d(\xi)$ \\
\hline $1-m^{2}$ & 0 & $2-m^{2}$ & 0 & 1 & $c s(\xi)$ \\
\hline$\left(m^{4}-m^{2}\right)$ & 0 & $2 m^{2}-1$ & 0 & 1 & $d s(\xi)$ \\
\hline$\frac{1}{4}$ & 0 & $\frac{1-2 m^{2}}{2}$ & 0 & $\frac{1}{4}$ & $n s(\xi) \pm c s(\xi)$ \\
\hline$\frac{1-m^{2}}{4}$ & 0 & $\frac{1+m^{2}}{2}$ & 0 & $\frac{1-m^{2}}{4}$ & $n s(\xi) \pm s c(\xi)$ \\
\hline$\frac{m^{2}}{4}$ & 0 & $\frac{m^{2}-2}{2}$ & 0 & $\frac{1}{4}$ & $n s(\xi) \pm d s(\xi)$ \\
\hline$\frac{m^{2}}{4}$ & 0 & $\frac{m^{2}-2}{2}$ & 0 & $\frac{m^{2}}{4}$ & $\frac{d n(\xi)}{\sqrt{1-m^{2}} \operatorname{sn}(\xi) \pm c n(\xi)}$ \\
\hline$\underline{\left(1-m^{2}\right)^{2}}$ & 0 & $\frac{1+m^{2}}{2}$ & 0 & $-\frac{1}{4}$ & $m \cdot c n(\xi) \pm d n(\xi)$ \\
\hline $\begin{array}{l}-4 \\
\frac{1}{4}\end{array}$ & 0 & $\frac{1-2 m^{2}}{2}$ & 0 & $\frac{1}{4}^{4}$ & $\frac{\operatorname{sn}(\xi)}{1+\cos (\xi)}$ \\
\hline $\begin{array}{c}4 \\
m^{2} \\
\end{array}$ & 0 & $\frac{1-2 m^{2}}{2}$ & 0 & $\frac{4}{1}$ & $\begin{array}{c}1 \pm c n(\xi) \\
d n(\xi) \\
\end{array}$ \\
\hline 4 & 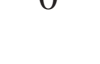 & & & $\overline{4 m^{2}}$ & $\sqrt{\frac{1-m^{2}}{m^{2}}} \pm c n(\xi)$ \\
\hline$\frac{1}{4}$ & 0 & $\frac{m^{2}-2}{2}$ & 0 & $\frac{m^{2}}{4}$ & $\frac{m^{2} n(\xi)}{1 \pm d n(\xi)}$ \\
\hline$\frac{m^{2}-1}{4}$ & 0 & $\frac{1+m^{2}}{2}$ & 0 & $\frac{1-m^{2}}{4}$ & $\frac{d n(\xi)}{1+m \cdot \operatorname{sn}(\xi)}$ \\
\hline$\frac{1-m^{2}}{4}$ & 0 & $\frac{1+m^{2}}{2}$ & 0 & $\frac{1-m^{2}}{4}$ & $\begin{array}{l}\frac{1 \pm m \cdot n(\xi)}{c n(\xi)} \\
\frac{1 \pm \operatorname{sn}(\xi)}{1 \xi}\end{array}$ \\
\hline$\frac{1}{4}$ & 0 & $\frac{1+m^{2}}{2}$ & 0 & $\frac{\left(1-m^{2}\right)^{2}}{4}$ & $s n(\xi)$ \\
\hline $\begin{array}{l}4 \\
\frac{1}{4}\end{array}$ & 0 & $\frac{2-m^{2}}{2}$ & 0 & $\begin{array}{c}4 \\
\underline{m^{4}}\end{array}$ & $\begin{array}{l}\operatorname{cn}(\xi) \pm d n(\xi) \\
\operatorname{cn}(\xi)\end{array}$ \\
\hline$\overline{4}$ & & & & $\overline{4}$ & $\sqrt{1-m^{2}} \pm d n(\xi)$ \\
\hline 0 & 0 & 1 & $A_{2}$ & $A$ & $\left(A_{3}+A_{3} \tan ^{2}(\xi-c) \pm \sqrt{\tan ^{2}(\xi-c)\left(1+\tan ^{2}(\xi-c)\right)\left(A_{3}^{2}+4 A_{4}\right)}\right)$ \\
\hline 0 & 0 & -1 & $A_{3}$ & $A_{4}$ & $\left(\frac{A_{3}^{2}-4 A_{4} \tan ^{2}(\xi-c)}{2}\right)$ \\
\hline 0 & 0 & 0 & $A_{3}$ & $A_{4}$ & $\frac{4 A_{3}}{A_{3}^{2} \xi^{2}-2 A_{3}^{2} c \xi+c^{2} A_{3}^{2}-4 A_{4}}$ \\
\hline 0 & $\mathrm{k}$ & $-2 k$ & $\mathrm{k}$ & 0 & $\tanh \left(\frac{\sqrt{k}}{2}(\xi-c)\right)$ \\
\hline 0 & 0 & $A_{2}$ & $A_{3}$ & $A_{4}$ & $4 A_{2} e^{( \pm \sqrt{ }}$ \\
\hline$-\mathrm{k}$ & $-k$ & 0 & 1.5 & 114 & 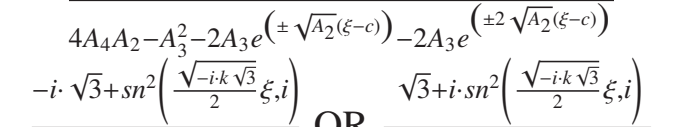 \\
\hline & ${ }^{-} \mathrm{n}$ & 0 & $\mathbf{K}$ & $\mathbf{K}$ & $\overline{i \cdot \sqrt{3}+\operatorname{sn}^{2}\left(\frac{\sqrt{-i \cdot k \sqrt{3}}}{2} \xi, i\right)} \quad \overline{-\sqrt{3}+i \cdot \operatorname{sn}^{2}\left(\frac{\sqrt{-i \cdot k \sqrt{3}}}{2} \xi, i\right)}$ \\
\hline 0 & $A_{1}$ & 0 & $A_{3}$ & 0 & $\frac{\sqrt{-A_{3} A_{1}}}{A_{3}}\left(-1+s n^{2}\left(\frac{\sqrt{2}}{2} \sqrt[4]{-A_{3} A_{1}} \xi, \frac{\sqrt{2}}{2}\right)\right)$ \\
\hline 0 & $-2 k^{2}$ & $-k^{2}$ & $k^{2}$ & 0 & $-1+s n^{2}\left(\frac{\sqrt{3}}{2} k \xi, \frac{\sqrt{3}}{3}\right)$ \\
\hline
\end{tabular}




\section{Appendix B}

Jacobi elliptic functions degenerate into hyperbolic functions

when $m \rightarrow 1$ :

$\operatorname{sn}(\xi) \rightarrow \tanh (\xi), \operatorname{cn}(\xi) \rightarrow \operatorname{sech}(\xi), \operatorname{dn}(\xi) \rightarrow \operatorname{sech}(\xi), \operatorname{sc}(\xi) \rightarrow \sinh (\xi)$,

$\operatorname{sd}(\xi) \rightarrow \sinh (\xi), c d \rightarrow 1, n s(\xi) \rightarrow \operatorname{coth}(\xi), n c(\xi) \rightarrow \cosh (\xi)$,

$n d(\xi) \rightarrow \cosh (\xi), \operatorname{cs}(\xi) \rightarrow \operatorname{csch}(\xi), d s(\xi) \rightarrow \operatorname{csch}(\xi), d c(\xi) \rightarrow 1$

Jacobi elliptic functions degenerate into trigonometric functions

when $m \rightarrow 0$ :

$\operatorname{sn}(\xi) \rightarrow \sin (\xi), \operatorname{cn}(\xi) \rightarrow \cos (\xi), d n(\xi) \rightarrow 1, s c(\xi) \rightarrow \tan (\xi)$,

$\operatorname{sd}(\xi) \rightarrow \sin (\xi), c d(\xi) \rightarrow \cos (\xi), n s(\xi) \rightarrow \csc (\xi), n c(\xi) \rightarrow \sec (\xi)$,

$n d(\xi) \rightarrow 1, c s(\xi) \rightarrow \cot (\xi), d s(\xi) \rightarrow \csc (\xi), d c(\xi) \rightarrow \sec (\xi)$.

\section{Copyrights}

Copyright for this article is retained by the author(s), with first publication rights granted to the journal.

This is an open-access article distributed under the terms and conditions of the Creative Commons Attribution license (http://creativecommons.org/licenses/by/3.0/). 